\title{
Experimental determination of representative elementary volume of sands using X-ray computed tomography
}

\author{
O. Al Hattamleh ${ }^{1}$, M. Razavi ${ }^{2}$ \& B. Muhunthan ${ }^{3}$ \\ ${ }^{1}$ Civil Engineering Department, The Hashemite University, Jordan \\ ${ }^{2}$ Department of Mineral Engineering, \\ New Mexico Institute of Mining and Technology, USA \\ ${ }^{3}$ Department of Civil and Environmental Engineering, \\ Washington State University, USA
}

\begin{abstract}
The concept of a representative elementary volume (REV) provides an effective means of developing macroscopic measures in the description of granular materials. However, due to the difficulties associated with the measurement and characterization of granular microstructure the existence and size of an REV has remained largely conjectural. This study presents a systematic method to examine the characteristics of the REV using X-ray computed tomography images. The 3-D images of Silica sand, and Ottawa sand have been characterized using advanced image processing techniques. The porosity variation of Silica sand and Ottawa sand showed three characteristic regions: an initial fluctuation region due to microscopic variations, a constant plateau region, and a region with a monotonic increase/decrease due to heterogeneity. The results show that for Silica sand composed mainly of elongated particles the REV is between 5 to 11 times of D50 and for Ottawa sand composed mainly of subrounded particles is between 9 to 16 times of D50.
\end{abstract}

Keywords: representative elementary volume (REV), porosity, X-ray computed tomography (X-ray CT), 3D image processing.

\section{Introduction}

The methods of continuum mechanics have provided an effective means of predicting the behavior of the collection of a large number of elements. The 
fundamental continuum hypothesis is that the behavior of many physical elements is essentially the same as if they were perfectly continuous. Physical quantities such as mass and density, associated with individual elements contained within a representative elementary volume (REV) are regarded as being spread over the volume instead of being concentrated on each particle or element. Macroscopic variables are defined typically as averages of microscopic variables over a REV. Literature is jamming of many recent treatises used the above argument [1-6], however, the difficulties associated with the measurement and the characterization of granular microstructure had prevented the identification of the size of REV in real media. In the absence of experimental measurements, the minimum dimensions of the REV are set by the grain size with the best guess being the REV is between 100 to 1000 grain diameters. For sandy soils, a REV with a radius of 10 to 20 grain diameters appears to have been adequate for obtaining well defined average for applications in ground water flow [7]. Customarily, the grain diameter of sand is measured using sieve analysis. The classifications of grain size measurement in sand in relation to other physical measurement are shown in table 1 [8].

Table 1: Classification of soil size in relation to other physical measurements [13].

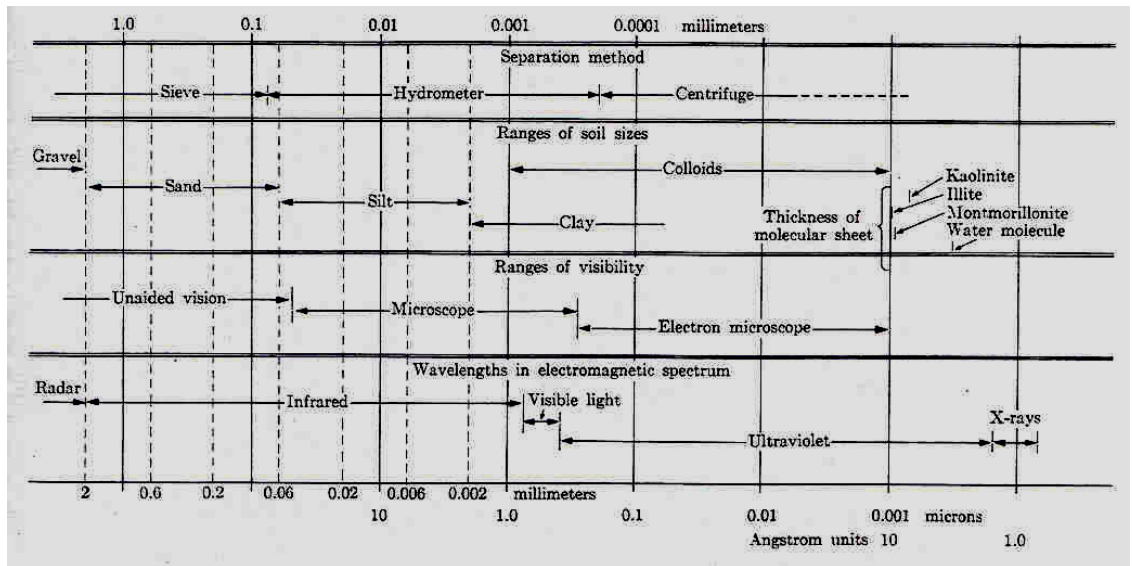

This study makes use of the current advances in microstructure characterization to accurately quantify the characteristics of REV of sands. High resolution X-ray computed tomography is used to obtain $3 \mathrm{D}$ images. These images are post processed using robust algorithms, and the variation of the porosity within a spherical volume element is studied.

\subsection{X-ray computed tomography}

X-ray computed tomography (X-ray CT) is a nondestructive technique that provides 3-D images of density distribution of specimens. Figure 1 shows the different parts of an industrial X-Ray CT system. Its main components include a 


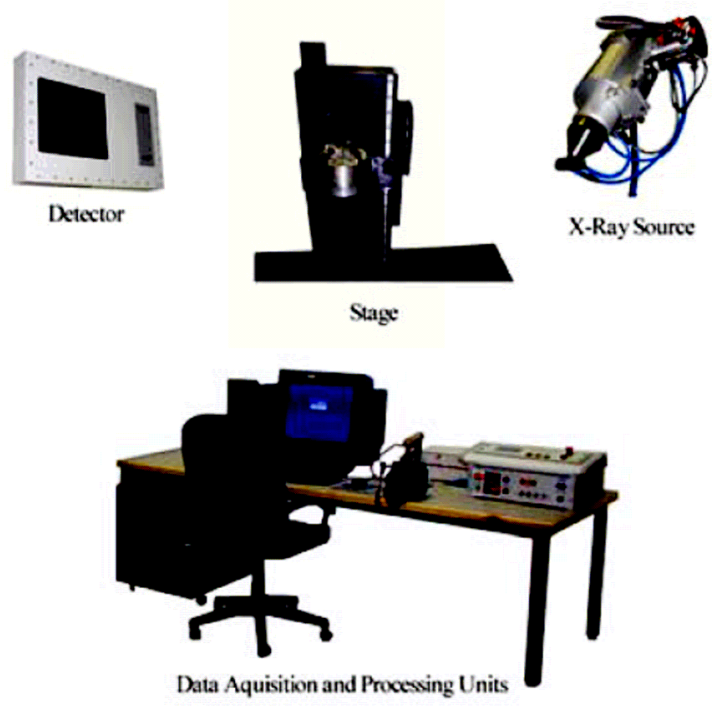

Figure 1: $\quad$ Main components of an industrial X-ray CT system.

radiation source of X-ray, a sensitive detector to $\mathrm{X}$-ray, a precision rotary stage, a data acquisition unit, and a processing unit. The specimen is placed on the rotary stage between the X-ray source and detector. The stage rotates in several small steps to complete a full circle of rotation. The intensity of the X-ray on the detector or the projected image of the specimen on the detector at each step is saved as a digital image or a digital radiograph. The CT image slices are reconstructed from the digital radiographs [9]. The density distribution across the cross section of slices are reconstructed from the set of projection images (radiographs) using Radon transformation. This transformation is fundamental to $\mathrm{X}$-ray CT reconstruction algorithms [9]. Spatial resolution, a measure of the ability of the X-ray CT system to separate and distinguish the minute details, depends on many factors such as the X-ray tube type and detector resolution. A CT image is the representation of the distribution of the point-by-point linear attenuation coefficients within the slice. These coefficients depend on the physical density of the material, its effective atomic number, and the X-ray beam energy. X-ray CT image is highly sensitive to differences in density; a difference of less than $1 \%$ in density is sufficient to separate objects. Voxels, the boxshaped volumes defined by the area of the pixel and the height of the slice thickness, form the 3-D CT image [10].

CT slices are stacked at equal distances on top of each other and the voxel value of the space between two successive reconstructed slices is linearly interpolated. The X-ray CT images are gray scale images in which normally white and black colors correspond to the highest and lowest densities, respectively. Figure 2(a) shows a two-dimensional CT slice of silica sand and 


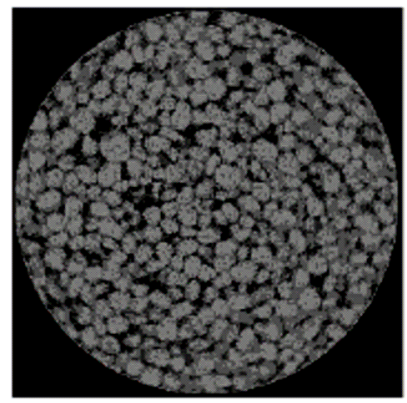

(a)

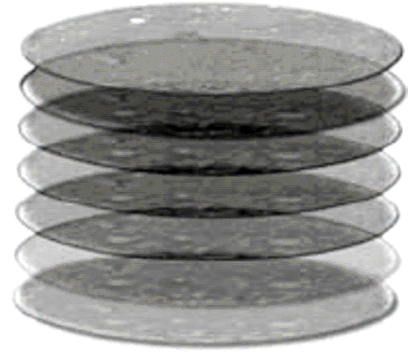

(b)

Figure 2: $\quad$ (a) A 2-D CT slice of silica sand; (b) A pile of 2-D CT slices to generate a 3-D CT image.

Fig. 2(b) shows how a three-dimensional CT images is generated by a pile of two-dimensional CT slices.

The CT scans for this study were done at the Washington State University high resolution X-ray computed tomography laboratory (www.waxct.wsu.edu). The facility consists of a flat panel amorphous silicon high-resolution computed tomography or FlashCT. FlashCT at WSU is a hybrid system with two different X-ray sources, $420 \mathrm{keV}$, and $225 \mathrm{keV}$ micro-focus housed within a single chamber. Micro-focus X-ray source takes advantage of an electromagnetic field and a vacuum pump around the X-ray outlet to reduce the focal spot size. Minimum focal spot size of this source is $5 \mu \mathrm{m}$ at 60 watts, which provides a spatial resolution down to $5 \mu \mathrm{m}$, and maximum operating energy of this tube is $225 \mathrm{keV}$. The micro-focus source was used for this study to obtain the highest possible resolution.

\section{Materials and methods}

Specimens were prepared from Ottawa and silica sands. The shape of the particles of Ottawa sand is composed mainly of subrounded particles, and that of silica sand is composed mainly of elongated particles. Specific gravity of Ottawa and silica sands are 2.65 and 2.7 respectively.

Table 2: $\quad$ Scan parameters of the specimens.

\begin{tabular}{|c|c|c|c|c||}
\hline Specimen & $E, \mathrm{keV}$ & $\mathrm{I}, \mathrm{m} A$ & Image Resolution, $\mu \mathrm{m} /$ pixel & Image Size, voxel \\
\hline SS-1 & 160 & 0.284 & 40.0 & $675 \times 671 \times 672$ \\
\hline SS-2 & 160 & 0.284 & 39.7 & $680 \times 679 \times 500$ \\
\hline SS-3 & 160 & 0.284 & 39.8 & $675 \times 678 \times 500$ \\
\hline OS-1 & 160 & 0.284 & 39.5 & $683 \times 679 \times 500$ \\
\hline OS-3 & 160 & 0.284 & 39.5 & $679 \times 683 \times 500$ \\
\hline OS-4 & 160 & 0.284 & 39.6 & $682 \times 682 \times 500$ \\
\hline
\end{tabular}


Six cylindrical specimens of both dry silica sand (SS-1 to SS-3) and Ottawa sand (OS-1 to OS-3) with different porosities were prepared in the laboratory. They were compacted in five layers by tamping on the sides of the plastic mold. All of the specimens were scanned using X-Ray CT following [11], ASTM E 1441-00, and their 3D images obtained. Table 2 shows the CT scan parameters, image resolution, and number of voxels for each specimen in the study.

\section{REV and image characterization}

An interactive computer program, M-REV, was developed in MATLAB environment to perform the analyses on the $3 \mathrm{D}$ CT images. First, the 3D CT image is read and stored as a 3D array so that the reconstructed CT slices show the top view of the specimen. If necessary the gray scale values of the voxels can be rescaled to spread the histogram of the gray scale values between 0 and 255. This is called histogram equalization for intensity adjustment. In most cases, CT slices contain some noise on the image background, which tends to propagate a significant error in processing. User can specify the outer boundaries of the specimen in the program to remove background noise.

In the next step, the image is converted to a logical image (black and white image or BW image) using a threshold value. In this study, the method proposed by [12], which chooses the threshold to minimize the interclass variance of the black and white pixels, was applied to find the best threshold to convert the image to a logical image. It is noted, however, that the user can manually choose a desired threshold between 0 and 255 to separate the features by looking at the image histogram and visual inspection. However, the use of a threshold alone does not separate the grain boundaries very well. Therefore, another advanced image processing technique called watershed transform $[12,13]$ with gradient is applied to segment or separate the contact points of particles. The watershed transform applies the same idea as in geography for segmentation of the gray scale images. Watershed ridgeline is the line, which separates the two connected objects. In order to apply watershed transformation to binary images, first a transformation of the distance from every pixel to the nearest nonzero valued pixel is calculated. Once the distance transformation of the image is determined, then watershed transformation is applied. In some cases, even with the use of watershed transform the boundaries of the particles may not be clear. For such cases, application of a gradient prior to using the watershed transformation is recommended. In the gradient method, the image is filtered by a $3 \times 3$ Sobel mask, which approximates vertical or horizontal gradients of the image [13]. In case of over-segmentation due to watershed transform in which many grains have been segmented around the boundaries and inside, the method of regional minima is applied to remove the unnecessary segmentation within the grains [13].

The REV program chooses a spherical volume element whose center can be fixed anywhere within the specimen (fig. 3). Once the location of the center is fixed, the radius of the sphere is increased from zero to its maximum limited by the specimen boundary. The variation of porosity with the radius of volume element is plotted for each incremental step of the spherical radius. 
150 Computational Methods and Experiments in Materials Characterisation IV

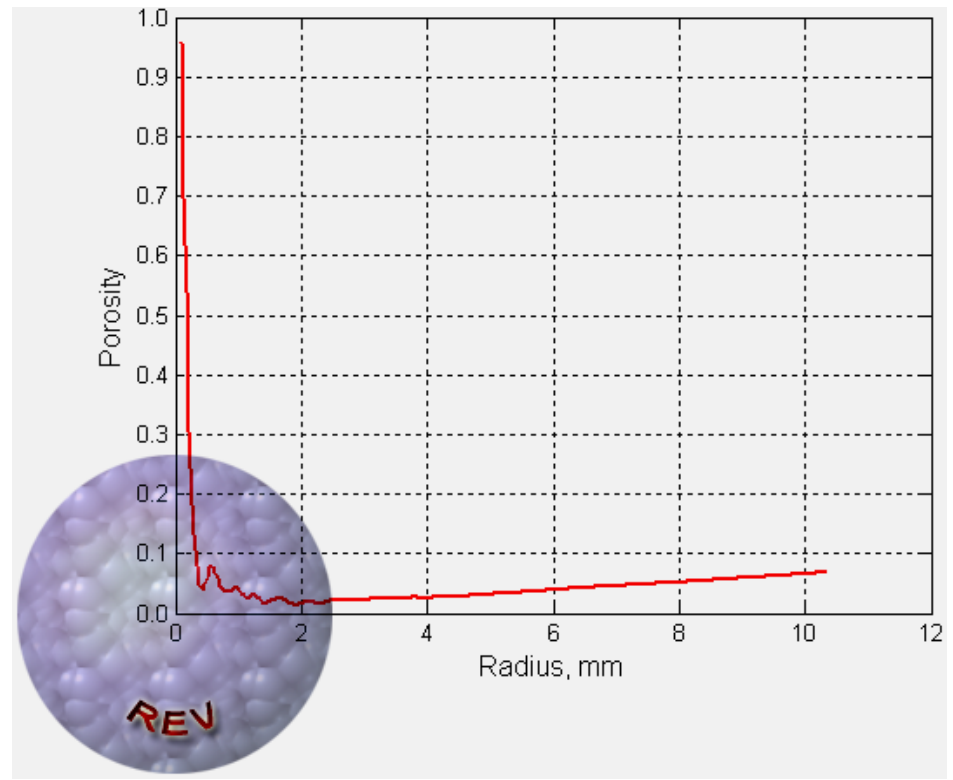

Figure 3: A sample plot of variations of porosity versus the radius of the spherical volume element.

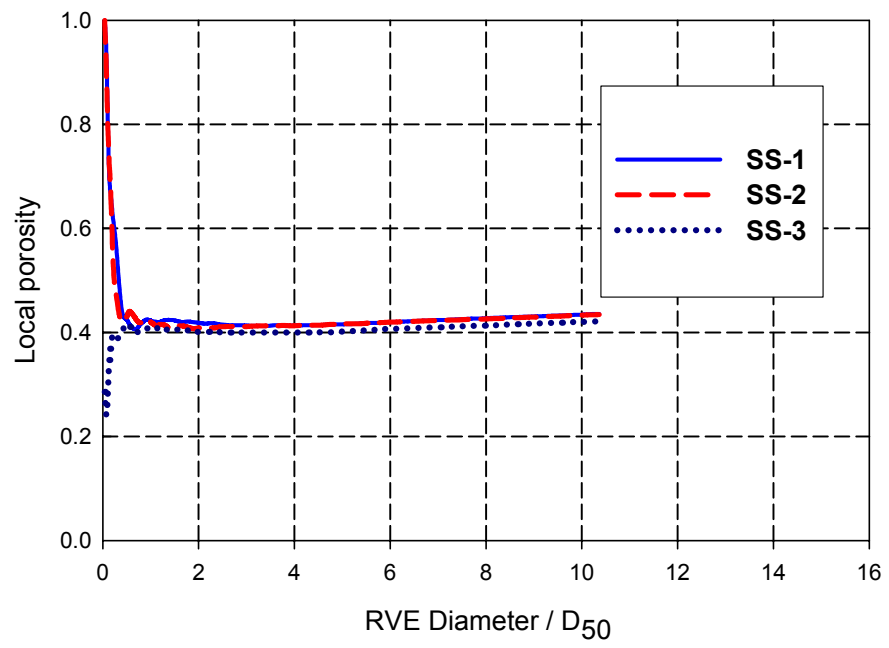

Figure 4: $\quad$ Normalized REV range to $\mathrm{D}_{50}$ for silica sand specimens.

In REV program, the user can examine the images from three different viewpoints; top, front, and right. The user can zoom (in or out) the images, find the location of each plane, the gray scale value of any voxel, and distance 
between different objects on images. The output results are saved in MATLAB, and can also be exported to spreadsheet.

\section{Results and discussion}

The variation of porosity, $n=V_{V} / V_{T}$ where $V_{v}$ the volume of voids and $V_{T}$ the total volume, versus the normalized radius of the spherical volume element with respect to main grain diameter, $\mathrm{D}_{50}$, for the three silica sand specimens is shown in Fig. 4. It can be seen that the variation of the porosity of all three specimens show three distinct regions; a segment with fluctuation part at the beginning, a constant segment in the middle and a monotonically increasing segment at the end. It is also evident that the boundaries of the region are nearly the same in all of the specimens. The same trend is evident in the case of Ottawa sand (Fig. 5) although the region III in these two materials has both an increase and a decreasing trend.

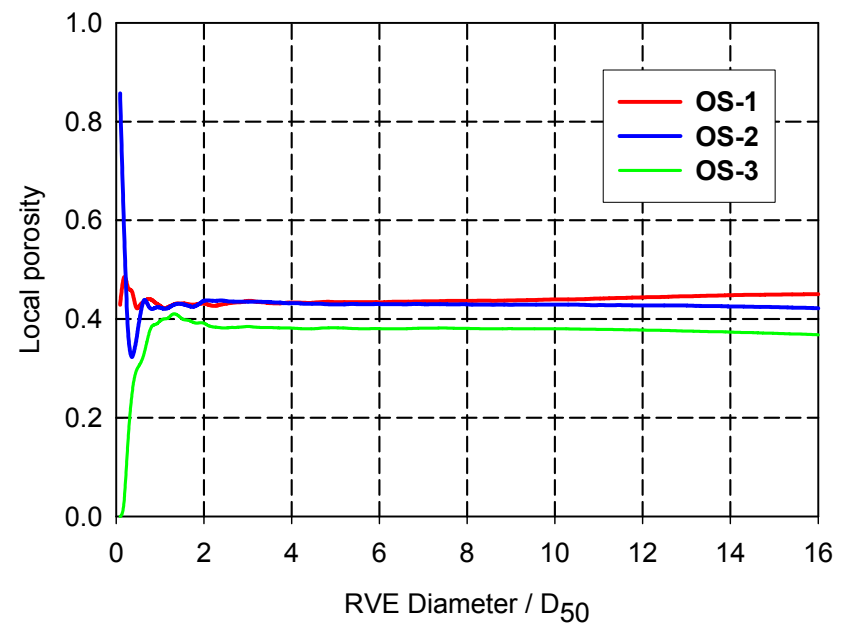

Figure 5: $\quad$ Normalized REV range to $\mathrm{D}_{50}$ for Ottawa sand specimens.

Figure 6 shows the plot of variation of porosity respect to the normalized radius of the spherical volume element for two selected specimens of silica sand (SS-3) and Ottawa sand OS-1). It can be seen that the REV radius for Ottawa sand is formed with more grains with a diameter of $D_{50}$. The ranges of representative elementary volume radius for all specimens are summarized in table 3. Comparison those ranges with $\mathrm{D}_{50}$ of sand specimens show that the ratio of the REV radius to $D_{50}$ is about 5 to 11 times of for silica sand, and 9 to 16 times for Ottawa sand.

The last column of table 3 shows the comparison of the porosity obtained from image processing $\left(\mathrm{n}_{\mathrm{ip}}\right)$ and the laboratory measured values $\left(\mathrm{n}_{\mathrm{lab}}\right)$. The values compare very well. It is noted, however, that the relative error is much 
higher in Ottawa sand. This sand had finer grains and although the size of the finest grains of Ottawa sand specimen was more than the resolution of CT image less number of pixels is used to form the image in sands with finer grains for a given magnification. This problem tends to propagate the error in processing the images and results in a higher relative error. Smaller specimens with higher magnification will reduce such error but the processing is limited by available memory.

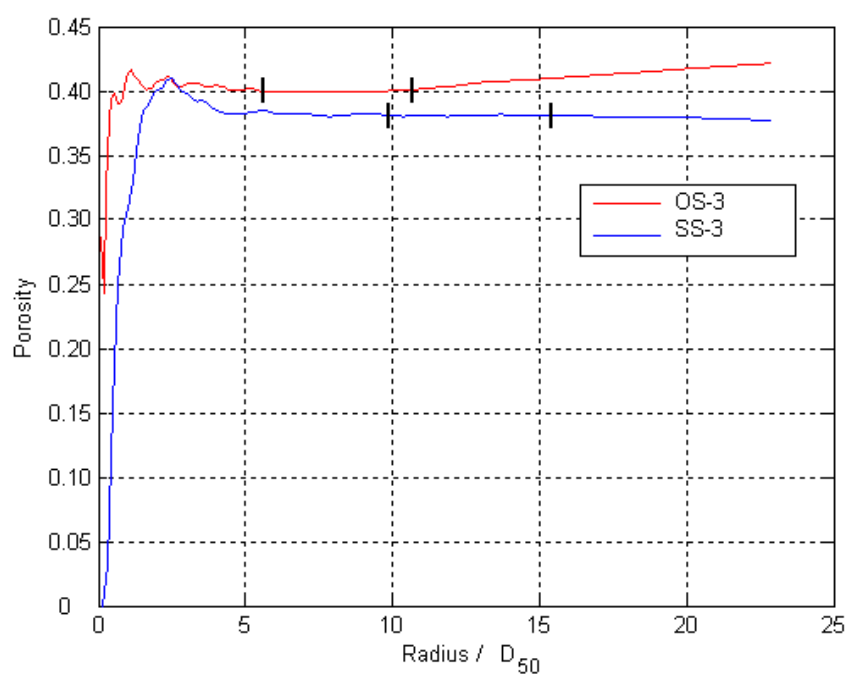

Figure 6: $\quad$ Normalized REV range to $\mathrm{D}_{50}$ for (SS-3) and (OS-1).

Table 3: $\quad$ REV radius and relative errors.

\begin{tabular}{|c|c|c|c|c|c|c||}
\hline \multirow{2}{*}{ Type } & Specimen & $\begin{array}{c}\mathbf{R}_{R E V} \text { Range, } \\
m m\end{array}$ & $\begin{array}{c}\mathbf{R}_{R E V} / \mathbf{D}_{50} \\
\text { Range }\end{array}$ & $n_{i p}$ & $n_{\text {lab }}$ & $\begin{array}{c}\% \\
\text { Error }\end{array}$ \\
\hline \multirow{3}{*}{$\begin{array}{c}\text { Silica } \\
\text { Sand }\end{array}$} & $\mathrm{SS}-1$ & {$[2.54,3.34]$} & {$[5.6,7.4]$} & $\mathbf{0 . 4 5 4}$ & $\mathbf{0 . 4 6 2}$ & $\mathbf{1 . 7 3}$ \\
\cline { 2 - 7 } & $\mathrm{SS}-2$ & {$[3.04,4.66]$} & {$[6.7,10.3]$} & $\mathbf{0 . 4 1 2}$ & $\mathbf{0 . 4 0 5}$ & -1.73 \\
\cline { 2 - 7 } & $\mathrm{SS}-3$ & {$[2.54,4.83]$} & {$[5.6,10.7]$} & $\mathbf{0 . 4 0 1}$ & $\mathbf{0 . 4 0 1}$ & $\mathbf{0 . 0 0}$ \\
\hline \multirow{3}{*}{$\begin{array}{c}\text { Ottawa } \\
\text { Sand }\end{array}$} & $\mathrm{OS}-1$ & {$[2.63,3.80]$} & {$[9.9,15.4]$} & $\mathbf{0 . 3 7 9}$ & $\mathbf{0 . 4 1 9}$ & $\mathbf{9 . 5 5}$ \\
\cline { 2 - 7 } & $\mathbf{O S}-2$ & {$[2.44,3.64]$} & {$[9.9,14.8]$} & $\mathbf{0 . 4 2 9}$ & $\mathbf{0 . 4 1 2}$ & -4.13 \\
\cline { 2 - 7 } & $\mathbf{O S}-3$ & {$[2.23,2.73]$} & {$[9.1,11.1]$} & $\mathbf{0 . 4 3 5}$ & $\mathbf{0 . 4 5 1}$ & $\mathbf{3 . 5 5}$ \\
\hline
\end{tabular}

\section{Conclusions}

This study presents a systematic technique to determine the size RVE of granular materials. It makes use of the X-ray computed tomography imaging techniques to determine the RVE for almost any granular material. 3-D CT images of Silica and Ottawa sands specimens, compacted in small cylindrical molds, were obtained. A 3-D interactive image processing program was developed to process the 3-D CT images to determine the local porosity variation. Comparing the 
local porosity variation obtained from the X-ray computed tomography imaging techniques with an average porosity of the sample obtained in the conventional laboratory tests enable us to determine the RVE. The REV radius for silica sand composed mainly of elongated particles is between 5 to 11 times of $\mathrm{D}_{50}$, and for Ottawa sand composed mainly of subrounded particles is between 9 to 16 times of $\mathrm{D}_{50}$.

\section{References}

[1] Mang, H. A. Eberhardsteiner, J., Hellmich, C., Hofstetter, K., Jäger, A., Lackner, R., Meinhard, K., Müllner, H.W., Pichler, Pichler, C. B., Reihsner, R., Stürzenbecher, R., \& Zeiml, M., Computational mechanics of materials and structures, Engineering Structures, doi:10.1016/ j.engstruct.2009.01.005

[2] Scholtès, L., Chareyre, B. Nicot, F. \& Darve, F., Micromechanics of granular materials with capillary effects, International Journal of Engineering Science, 47(1), pp. 64-75, 2009.

[3] Andrade, J.E. \& Tu, X., Multiscale framework for behavior prediction in granular media, Mechanics of Materials, doi:10.1016/ j.mechmat.2008.12.005

[4] Chen, K-C. \& Lan, J-Y., Micromorphic modeling of granular dynamics, International Journal of Solids and Structures, doi:10.1016/ j.ijsolstr.2008.11.022.

[5] Alonso-Marroquín, F., Mühlhaus, H.B. \& Herrmann, H.J., Micromechanical investigation of granular ratcheting using a discrete model of polygonal particles, Particuology, 6 (6), pp. 390-403, 2008.

[6] Maalej, Y., Dormieux, L., \& Sanahuja, J., Micromechanical approach to the failure criterion of granular media, European Journal of Mechanics A/Solids, doi:10.1016/j.euromechsol.2008.10.010.

[7] Charbeaneau, R. J., Groundwater Hydraulics and Pollutant Transport, Prentice Hall, NY, 2000.

[8] Scott, R.F., Principles of Soil Mechanics, Addison Wesely publishing company, Reading, Massachusetts, USA, 1963.

[9] Kak, A. C., \& Slaney, M., Principle of Computerized Tomographic Imaging, Siam, Philadelphia, 2001

[10] Dennis, M. J., Industrial Computed Tomography. Reprinted from Metals Handbook, 17, 9th Edition, pp.358-386, 1989.

[11] Annual Book of ASTM Standards, Nondestructive Testing, Vol. 03.03, 2004.

[12] Otsu, N., A Threshold Selection Method from Gray Level Histograms, IEEE, Trans. Systems, Man and Cybernetics, 9, pp.62-66, 1979.

[13] Gonzales, R. C., Woods, R. E., \& Eddins, S. L., Digital Image Processing using Matlab, Prentice Hall, Upper Saddle River, NJ 07458, 2004. 